\title{
Mapping of Self-organization Properties and Non-functional Requirements in Smart Grids ${ }^{\star}$
}

\author{
Sebastian Lehnhoff ${ }^{1}$, Sebastian Rohjans ${ }^{1}$, Richard Holzer ${ }^{2}$, \\ Florian Niedermeier ${ }^{2}$, and Hermann de Meer $^{2}$ \\ 1 OFFIS - Institute for Information Technology, Escherweg 2, 26121 Oldenburg, \\ Germany \\ firstname.lastname@offis.de \\ 2 Faculty of Computer Science and Mathematics, University of Passau, Innstraße 43, \\ 94032 Passau, Germany \\ firstname.lastname@uni-passau.de
}

\begin{abstract}
Future electrical power networks will be composed of large collections of autonomous components. Self-organization is an organizational concept that promises robust systems with the ability to adapt themselves to system perturbations and failures and thus may yield highly robust systems with the ability to scale freely to almost any size. In this position paper the authors describe the well-established process of use case based derivation of non-functional requirements in energy systems and propose a mapping strategy for aligning properties of selforganizing systems with the ICT- and automation system requirements. It is the strong belief of the authors that such a mapping will be a key factor in creating acceptance of and establishing self-organization in the domain of electrical energy systems.
\end{abstract}

Keywords: Smart Grid, Self-organization, Quality of Service, Quantitative Measures.

\section{Introduction}

In order to assess the requirements for ICT- and automation systems that have to support and enable a Smart Grid composed of large collections of autonomous components to perform appropriate functions a use case based requirements engineering methodology can be applied based on IEC PAS 62559 [1. Here, use cases are established for the purpose of identifying how a system should behave in relation to its actors and components in order to ensure a specified task or function e.g. in due time, with the necessary precision, while meeting certain service level agreements etc. Thus, use cases may document non-functional requirements for usage scenarios of future electrical power networks and support technology decisions in order to implement extensive and complex Smart Grids.

\footnotetext{
* This research is partially supported by SOCIONICAL (FP7, ICT-2007-3-231288), by All4Green (FP7, ICT-2011-7-288674) and by the NoE EINS (FP7, ICT-2011-7288021).
} 
In this position paper we propose a mapping strategy for aligning properties of self-organizing systems with the ICT- and automation system Quality of Service (QoS) requirements.

\section{Use Case Based Requirements Engineering in Smart Grids}

In order to derive what kind of functions to implement and what kind of systems to build for executing a future Smart Grid Use Case a number of questions need to be addressed [2]. Functional issues may address the system's flexibility for e.g. providing some kind of power reserve for load balancing, or the system's capability to detect certain phenomena that may be characteristic of a stability issue (if these are part of the given use case). However, for the development of a proper supporting ICT-system non-functional requirements may be more relevant and address issues like safety, security, performance or accuracy of certain measurements. In order to manage these kinds of issues and questions the methodology utilizes a reference architecture to identify participating system components and their interfaces to each other. A properly defined use case should then be able to identify relevant actors and components consistently from within this reference architecture and specify their interactions with each other. The European Union has issued a mandate (M/490) for the standardization of Smart Grid functionalities and use cases [3] to three European standards organizations (CEN, CENELEC and ETSI) to develop a common framework for representing functional information data flows between power system domains and their relevant subdomains as well as their architectures (the Smart Grid Architecture Model - SGAM) 4]. Additionally, a sustainable process and method is developed for use case specification and formalization allowing stakeholder interactions, gap analysis and (for the scope of this paper most important) the derivation of non-functional requirements necessary for the successful execution of a given task.

\subsection{The Smart Grid Reference Architecture}

The ISO/IEC 42010 defines a reference architecture as a description of a system's structure in terms of interactions between its element types and their environment [5]. The SGAM is the reference architecture for describing the Smart Grid and especially ICT- and automation systems within this domain. Starting from a contextual "component layer" spanning the power system domains in terms of the energy conversion chain and its equipment against the hierarchical levels (zones) of power system management (see bottom layer in Figure 1). The purpose of this layer is to emphasize the physical distribution of all participating components including actors, applications, power system equipment (at process and field level), protection and remote-control devices, communication infrastructure as well as any kind of computing systems. 


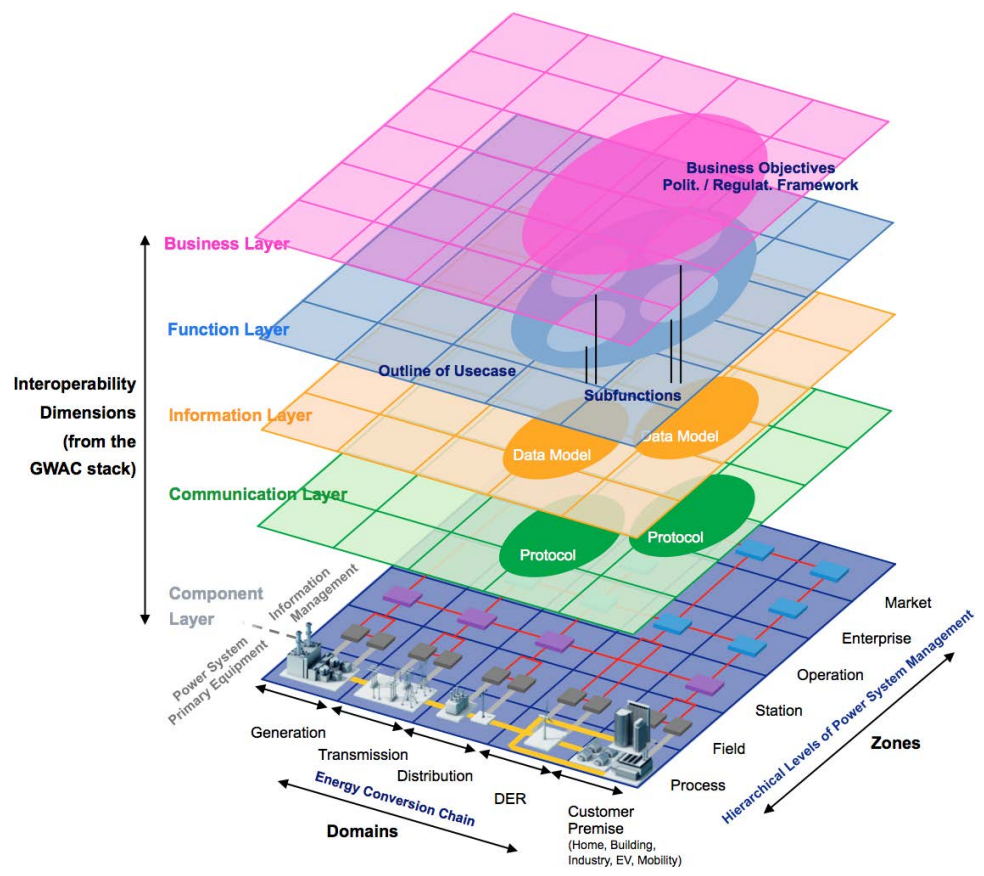

Fig. 1. Smart Grid Architecture Model (SGAM, 6])

For the development of interoperable Smart Grid architectures the component layer is extended by additional interoperability layers derived from the highlevel categorization approach developed by the GridWise Architecture Council 7. (GWAC stack, see Figure 1). Its layers comprise a vertical cross-section of the degrees of interoperation necessary to enable various interactions and transactions within a Smart Grid. Basic functionality (e.g. interaction with field equipment, transcoding and transmitting data) is confined to the lower component and communication layers. Standards for data and information modeling and exchange are defined on the information layer while the top functional and business layers deal with business functionality. As the functions, capabilities and participating actors increase in terms of complexity, sophistication and number, respectively, more layers of the GWAC stack are utilized in order to achieve the desired interoperable results. Thus, each layer typically depends upon and is enabled (through the definition of well known interfaces) by the layers below it.

\subsection{Methodology for Use Case Based Requirements Engineering in Energy Systems}

Within this framework for a sustainable development process, which is based on the IEC PAS 62559, use cases can be formally described detailing functional and performance requirements in order to assess its applicability and (if so) 
appropriate standards and technologies [8] 9]. Thus, the relevant contents of a use case are actor specifications, assumptions about the use case scenario, contracts and preconditions that exist between the actors and are necessary to set the use case into motion, triggering events and a numbered list of events detailing the underlying control and communication processes. UML-based class and sequence diagrams are used to describe the actor setup and how processes operate with one another and in what order. For the mapping of the use case onto the SGAM the following steps are recommended [6] starting from the function layer and "drilling down" to the components, communication and information layers:

1. Identify those domains and zones, which are affected by the use case

2. Outline the coverage of the use case in the smart grid plane

3. Distribute sub-functions or services of the use case to appropriate locations in the smart grid plane

4. Continue down to the next layers while verifying that all layers are consistent, there are no gaps and all entities are connected.

This process model allows the detailed specification of non-functional requirements for each step within the use case's sequence diagram. In order to provide a consistent classification and to enable a mapping of requirements on suitable standards and technologies the PAS 62559 provides comprehensive check lists for non-functional requirements ([1], p. 57ff.) within the areas of Configuration, Quality of service, Security requirements, Data management issues, Constraints and other issues. The QoS-requirements do not only allow specifying what standards and protocols to use but also support the decisions regarding technology or (organization, management) algorithms in order to achieve the necessary performance, availability of the system (acceptable downtime, recovery, backup, rollback, etc.) the frequency of data exchanges, and the necessary flexibility for changes in a future Smart Grid interconnecting a vast number of sensors and actuators.

\section{Measures for Self-organizing Systems}

To this end self-organizing systems are to achieve necessary levels of service quality in complex large-scale technical systems while at the same time increasing adaptivity and robustness of the system through distribution of (critical) control. In the recent years some measures of self-organizing properties in complex systems have been developed [10, 11, [12]:

Autonomy: A measure for autonomy specifies the amount of external data needed to control the system.

Emergence: A measure for emergence indicates whether some global structures are induced by the local interactions between the entities.

Global state awareness: A measure for global state awareness specifies the amount of information of each single entity about the relevant global properties, such that the entities are able to make the right decisions to fulfill the overall goal of the system. 
Target orientation: A measure for target orientation specifies whether the overall goal of the system is satisfied.

Adaptivity: A measure for adaptivity specifies how good the system can react to changes in the environment, such that the overall goal is still satisfied.

Resilience: A measure for resilience describes how good the system can react to abnormal events in the system like malfunctioned nodes, attacks against some entities of the system or a break down of some entities.

Each of these 6 measures is time dependent and yields values in the real interval $[0,1]$, where the value 1 means that the property is fully satisfied in the current point in time, while the value 0 means that the property is not satisfied at all in the current point in time. Therefore each measure can be seen as a map $m: T \rightarrow[0,1]$ from the time space into the interval $[0,1]$. Averaging over time, this measure evaluates the whole system.

\subsection{Evaluation of QoS-Requirements of Smart Grid ICT- and Automation Systems}

Some of these measures used in self-organizing systems can be effortlessly applied for the evaluation of QoS-requirements of Smart Grid ICT- and automation systems. Since an analytical evaluation of the measures usually is too complex in large systems, approximation methods are needed. An evaluation of the measures can either be done by simulations or by measurements in a real system. The results can be used for the specification of design criteria in the engineering process of Smart Grids and for the optimization of existing Smart Grids.

To achieve this goal the following steps have to be performed:

1. Specify a use case including the corresponding list of QoS-requirements that have to be fulfilled.

2. Specify a micro-level model describing the entities and their interactions.

3. For each QoS-requirement specify the corresponding measure which can be used for the evaluation of the requirement.

4. Combine the measures of 3 , to get an overall measure for the evaluation of the QoS-requirements.

5. Choose an evaluation method (e.g. simulations, measurements in real systems, etc.).

6. Evaluate the measure for the Smart Grid for different system parameters.

7. Find the optimal values for the system parameters, which fulfill all requirements.

A micro-level model according to step 2 can easily be specified based on the SGAM. However, step 3 requires a semantic mapping from the IntelliGrid QoSontology on the afore mentioned properties.

\section{Conclusion and Future Work}

A mapping between self-organization properties and the use case requirements is needed in order to support explicit design decisions towards self-organizing 
systems in future Smart Grids. For selected use cases in Smart Grids we plan to evaluate different QoS requirements with the developed measures [11] [12] to extract design criteria and to optimize system parameters with respect to the specified requirements. We will report on the outcoming of this evaluation in upcoming publications.

\section{References}

1. International Electrotechnical Commission (IEC), Publicly available specification (pas) 62559 intelligrid methodology for developing requirements for energy systems (2008)

2. Rohjans, S., Daenekas, C., Uslar, M.: Requirements for smart grid ict architectures. In: 3rd IEEE PES Innovative Smart Grid Technologies (ISGT) Europe Conference (2012)

3. European Commission, Smart Grid Mandate - Standardization Mandate to European Standardisation Organisations (ESOs) to support European Smart Grid deployment. M/490 EN (2011)

4. Bruinenberg, J., Colton, L., Darmois, E., Dorn, J., Doyle, J., Elloumi, O., Englert, H., Forbes, R., Heiles, J., Hermans, P., Kuhnert, J., Rumph, F.J., Uslar, M., Wetterwald, P.: Smart grid coordination group technical report reference architecture for the smart grid version 1.0 (draft) (March 3, 2012). tech. rep. (2012)

5. ISO/IEC/IEEE, Systems and software engineering - architecture description, ISO/IEC/IEEE 42010:2011(E) (Revision of ISO/IEC 42010:2007 and IEEE Std 1471-2000), vol. 1, pp. 1-46 (2011)

6. CEN, CENELEC, ETSI, SGCP report on reference architecture for the smart grid v0.5 (for sg-cs sanity check) (January 25, 2012), tech. rep. (2012)

7. T. G. A. Council, GridWise Interoperability Context- Setting Framework (2008)

8. Uslar, M., Specht, M., Rohjans, S., Trefke, J., Gonzalez, J.M.: The Common Information Model CIM: IEC 61968/61970 and 62325 P Practical Introduction to the CIM. Springer (2012)

9. Wegmann, A., Genilloud, G.: The role of 'roles' in use case diagrams. EPFL-DSC DSC/2000/024, EPFL-DSC CH-1015 Lausanne (2000)

10. Auer, C., Wüchner, P., de Meer, H.: The degree of global-state awareness in selforganizing systems. In: Spyropoulos, T., Hummel, K.A. (eds.) IWSOS 2009. LNCS, vol. 5918, pp. 125-136. Springer, Heidelberg (2009)

11. Holzer, R., de Meer, H., Bettstetter, C.: On autonomy and emergence in selforganizing systems. In: Hummel, K.A., Sterbenz, J.P.G. (eds.) IWSOS 2008. LNCS, vol. 5343, pp. 157-169. Springer, Heidelberg (2008)

12. Holzer, R., de Meer, H.: Quantitative Modeling of Self-Organizing Properties. In: Spyropoulos, T., Hummel, K.A. (eds.) IWSOS 2009. LNCS, vol. 5918, pp. 149-161. Springer, Heidelberg (2009) 